\title{
Interactions between Adenylate Cyclase and the Yeast GTPase-Activating Protein IRA1
}

\author{
MARIJANE R. MITTS, JUDITH BRADSHAW-ROUSE, AND WARREN HEIDEMAN* \\ School of Pharmacy, University of Wisconsin, 425 North Charter Street, Madison, Wisconsin 53706
}

Received 13 March 1991/Accepted 31 May 1991

\begin{abstract}
The adenylate cyclase system of the yeast Saccharomyces cerevisiae contains many proteins, including the CYR1 polypeptide, which is responsible for catalyzing the formation of cyclic AMP from ATP, RAS1 and RAS2 polypeptides, which mediate stimulation of cyclic AMP synthesis by guanine nucleotides, and the yeast GTPase-activating protein analog IRA1. We have previously reported that adenylate cyclase is only peripherally bound to the yeast membrane. We have concluded that IRA1 is a strong candidate for a protein involved in anchoring adenylate cyclase to the membrane. We base this conclusion on the following criteria: (i) a disruption of the IRAI gene produced a mutant with very low membrane-associated levels of adenylate cyclase activity, (ii) membranes made from these mutants were incapable of binding adenylate cyclase in vitro, (iii) IRA1 antibodies inhibit binding of adenylate cyclase to the membrane, and (iv) IRA1 and adenylate cyclase comigrate on Sepharose $4 B$.
\end{abstract}

Ras proteins in the yeast Saccharomyces cerevisiae share many similarities to the mammalian $\mathrm{p} 21^{\text {ras }}$ proto-oncogene products. Both yeast and mammalian Ras proteins share structural homology with the other members of the large family of GTP-binding proteins that includes the $G$ proteins. Beyond the structural similarities, in both $S$. cerevisiae and mammals, Ras proteins play an important functional role in regulating cell division (1). The activity of Ras proteins is in turn regulated by a conserved family of proteins that inactivate Ras proteins. This family is represented by GTPaseactivating protein (GAP) (14) and neurofibromatosis type 1 $(3,22,23)$ in mammals and by IRA1 and IRA2 (inhibitory regulator of the Ras-cyclic AMP (cAMP) pathway) in $S$. cerevisiae (18-20). These proteins function in their respective systems by stimulating the GTPase activity intrinsic to Ras proteins, converting the active, GTP-bound Ras to the inactive, GDP-bound form (19-21). The effector for mammalian p $21^{\text {ras }}$ has not been identified; in yeast cells, however, the effector for Ras is adenylate cyclase. Thus, in yeast cells, inactivation of Ras by IRA1 and IRA2 appears necessary for reducing cAMP levels in response to nutritional limitation. We have recently reported that adenylate cyclase is peripherally bound to the membrane through an unidentified protein anchor (15). We now present evidence indicating that, in addition to its regulatory role, IRA1 has a structural role: anchoring adenylate cyclase to the membrane. Our results support a model in which both the effector for Ras and the terminator for the Ras signal are bound together.

\section{MATERIALS AND METHODS}

Strains. The yeast strains used are described in Table 1. Materials. Adenylate cyclase-grade $\left[\alpha{ }^{-32} \mathrm{P}\right] A T P(40 \mathrm{cpm} /$ pmol) was from Amersham, $\left[{ }^{3} \mathrm{H}\right] \mathrm{cAMP}(10,000 \mathrm{cpm}$ per assay) and $\mathrm{Na}^{125} \mathrm{I}$ were from New England Nuclear, and lectin affinity resins were from Sigma.

Media. Yeast cells were grown on either rich medium (YPD) containing $1 \%$ yeast extract, 2\% Bacto-peptone (Difco), and $2 \%$ glucose or minimal medium (SD) containing 6.7 $\mathrm{g}$ of yeast nitrogen base per liter, $2 \%$ glucose, and the

\footnotetext{
* Corresponding author.
}

appropriate factors to support the growth of auxotrophic strains.

Disruptions of the IRAI gene. The 1.05-kb BclI-PstI fragment of IRAI was inserted into the BamHI-PstI sites of pIC20R (13). The resulting plasmid was then digested with $X b a I$ and HindIII, and the 1.37-kb HindIII-XbaI fragment of IRAI was inserted. The resulting plasmid was then cut with HindIII, and the 1.24-kb HindIII fragment of YEp24 (2) containing the $U R A 3$ gene was inserted. The resulting plasmid contains the IRAI sequence from the Bcll site at position 2658 to the $X b a I$ site at position 5395 , with the URA3 gene and a short sequence of synthetic polylinker inserted in place of the region between the PstI site at position 3708 and the HindIII site at position 4026. This plasmid, pIKO, was used to disrupt the IRAI gene in strains HR125 and NW239C, producing strains TM101 and TM102, respectively, by one-step gene disruption (16). Plasmid pNR1 (18), provided by K. Tanaka, was used to disrupt the IRAI gene at position 8103 in strain HR125, resulting in mutant TM105. Positions are indicated according to the sequence reported by Tanaka et al. (18). Disruptions were confirmed by using the polymerase chain reaction and primers flanking the insertion site to amplify the region of genomic DNA disrupted by the marker.

Preparation of yeast particulate fractions. Yeast cells were grown to an optical density at $660 \mathrm{~nm}$ of approximately 2 to 4 . The cultures were centrifuged, and cells were disrupted by vortexing with glass beads in YMB buffer, containing $50 \mathrm{mM}$ 2- $N$-morpholinoethanesulfonic acid (MES; pH 6.0), $0.1 \mathrm{mM}$ EDTA, $0.1 \mathrm{mM} \mathrm{MgCl}_{2}, 1 \mathrm{mM}$ phenylmethylsulfonyl fluoride, and $50 \mu \mathrm{g}$ of leupeptin per $\mathrm{ml}$, as previously described (5). The homogenate was centrifuged in a Beckman J6 apparatus for $5 \mathrm{~min}$ at $1,000 \mathrm{rpm}(300 \times \mathrm{g})$ to remove nuclei and unbroken cells. The supernatant was then spun for 30 $\min$ at $14,000 \mathrm{rpm}(16,000 \times g)$ to separate particulate and soluble fractions. Both particulate and soluble fractions were stored in YMB at $-70^{\circ} \mathrm{C}$ until needed.

Reconstitution assays. Membranes from wild-type yeast strain $381 \mathrm{G}(17.6 \mathrm{mg})$ were extracted with $1.0 \mathrm{ml}$ of YMB containing $500 \mathrm{mM} \mathrm{NaCl}$ on ice for $30 \mathrm{~min}$ and centrifuged for $30 \mathrm{~min}$ at $14,000 \mathrm{rpm}(16,000 \times g)$ in an Eppendorf microcentrifuge, and the supernatant (extract) containing 
TABLE 1. Yeast strains

\begin{tabular}{|c|c|c|}
\hline Strain & Genotype & Source \\
\hline 381G (wild type) & MATa ade2-1 his4-580 lys2 trpl tyrl SUP4-3 cryR & L. Hartwell \\
\hline HR125 (wild type) & MATa leu2-3 leu2-112 ura3-52 trpl-1 his3-532 his4 & J. F. Casperson \\
\hline NW23-9C (cyrl-1) & MATa leu2-3 leu2-112 ura3-52 trpl-1 his3-532 his4 cyrl-1 cam & J. F. Casperson \\
\hline HR125::pAC2 (multicopy CYRI) & MATa leu2-3 leu2-112 ura3-52 trp1-1 his3-532 his4::YEp24:CYR1:URA3 & J. F. Casperson \\
\hline TC41-1 (cyrl) & MATa leu2-3 leu2-112 ura3-52 trpl-1 his3-532 his 4 cyrl::URA3 cam & W. Heideman \\
\hline $\begin{array}{l}\text { TM101 (IRAI disruption at } \\
\text { codon 1058) }\end{array}$ & MATa leu2-3 leu2-112 ura3-52 trpl-1 his3-532 his4 iral::URA3 & This work \\
\hline $\begin{array}{l}\text { TM102 (IRAI disruption at } \\
\text { codon } 1058, \text { cyrl) }\end{array}$ & $\begin{array}{l}\text { MATa leu2-3 leu2-112 ura3-52 trpl-1 his3-532 his } 4 \text { cyrl-1 cam } \\
\text { iral::URA3 }\end{array}$ & This work \\
\hline $\begin{array}{l}\text { TM105 (IRAI disruption at } \\
\text { codon } 2520)\end{array}$ & MATa leu2-3 leu2-112 ura3-52 trpl-1 his3-532 his4 iral::LEU2 & This work \\
\hline
\end{tabular}

adenylate cyclase activity was collected. Samples containing different amounts of extract (as indicated) were mixed with 5 $\mathrm{mg}$ of acceptor membranes prepared from $C Y R I$-deleted (cyrl) strains (with no adenylate cyclase activity). Reconstitution of adenylate cyclase to the acceptor membranes was initiated by diluting $\mathrm{NaCl} 10$-fold with reconstitution buffer (50 mM MES, $5 \mathrm{mM} \mathrm{MgCl}$, $\mathrm{pH}$ 6.0), and the mixture was incubated on ice for $30 \mathrm{~min}$. The membranes were collected by centrifugation, resuspended in $250 \mu$ l of reconstitution buffer, and assayed for adenylate cyclase activity in the presence of $\mathrm{Mn}^{2+}$. Values plotted represent specific binding, obtained by subtracting the activity of control samples containing no acceptor membranes (15). Acceptor membranes used were from cyrl strains with either a wild-type IRAI (TC41-1) or a cyrl strain carrying the IRAI disruption at codon 1058 (TM102).

Adenylate cyclase assay. Adenylate cyclase was assayed by a modification of the method of Salomon et al. (17) as described by Casperson et al. (4). Guanine nucleotides, where present, were at $10 \mu \mathrm{M}$; manganese was at $10 \mathrm{mM}$. Protein was assayed by the method of Lowry et al. (12).

B-Galactosidase/IRA1 fusion protein and preparation of antibodies. The 1.47-kb BamHI-XbaI fragment of IRAI was isolated and inserted into pUR288. This placed the IRAI gene fragment in frame at the carboxy-terminal end of $\beta$-galactosidase, allowing expression of amino acids 1127 to 1618. The resultant plasmid, pIC/D, was used to transform Escherichia coli JM105. Induction of cultures with isopropylthio- $\beta$-galactoside resulted in overexpression of a 170$\mathrm{kDa}$ protein in bacteria transformed with $\mathrm{pIC} / \mathrm{D}$. The protein was then partially purified by sodium dodecyl sulfate (SDS)polyacrylamide gel electrophoresis (PAGE), dialyzed into buffer (containing $20 \mathrm{mM}$ Tris [pH 7.4], $150 \mathrm{mM} \mathrm{NaCl}, 5 \mathrm{mM}$ $\mathrm{MgCl}_{2}, 1 \mathrm{mM}$ EDTA, and $1 \mathrm{mM}$ dithiothreitol), and used to immunize rabbits.

Western immunoblots. Protein samples (yeast membranes or column fractions) were separated on 4 or $6 \%$ polyacrylamide gels and transferred to nitrocellulose; the membranes were blocked with BLOTTO (5\% nonfat powdered milk in 50 mM Tris [pH 7.4]-150 mM NaCl-5 mM EDTA-0.01\% sodium azide- $0.05 \%$ Tween 20 [polyoxyethylene sorbitan monolaurate]) and developed with IRA1 antibody (1:600 dilution in BLOTTO). Immunoreactive proteins were visualized by the addition of ${ }^{125} \mathrm{I}$-labeled goat anti-rabbit antibody (specific activity, $2 \times 10^{6} \mathrm{cpm} / \mu \mathrm{g}$ ) and autoradiography of the washed membrane.

Lectin-agarose. Wild-type (HR125) membranes were detergent extracted (8) with YMB containing $2 \%$ taurocholate, $1 \mathrm{mM} \mathrm{Ca}^{2+}$, and $1 \mathrm{mM} \mathrm{Mn}^{2+}$, and samples of the extract were incubated with either Sepharose $4 B$, concanavalin A
(ConA) linked to Sepharose (ConA-Sepharose), or lentil lectin-agarose for $4 \mathrm{~h}$ at $4^{\circ} \mathrm{C}$. The resin samples were then washed with either YMB or YMB with $0.5 \mathrm{M} \mathrm{NaCl}$ added. Proteins were then eluted with $1 \%$ SDS-1\% $\beta$-mercaptoethanol-5 mM EDTA at $65^{\circ} \mathrm{C}$. Samples were run on a $4 \%$ SDS-polyacrylamide gel and prepared for Western blotting as described above.

\section{RESULTS}

Effects of IRAI disruptions on adenylate cyclase activity and subcellular localization. We created two disruptions of the IRAI coding region by inserting auxotrophic markers at positions 3708 and 8103 (codons 1058 and 2520; Fig. 1A). We then measured adenylate cyclase activity in membrane and cytosolic fractions from cells carrying either the wild-type or disrupted IRAI genes (Fig. 1B). The downstream disruption at codon 2520 (strain TM105) produced an increase in adenylate cyclase activity consistent with the other phenotypes reported for this disruption. Adenylate cyclase activity in the presence of $\mathrm{Mn}^{2+}$. ATP (a measure of catalytic activity of the enzyme independent of Ras stimulation) was slightly higher than the activity in the wild type (HR125). However, a more striking feature of this mutation was the substantial elevation of basal activity, as measured with guanosine $5^{\prime}-O$-(2-thiodiphosphate). This result is consistent with reports that this disruption raises cAMP levels and blocks the ability of IRA1 to accelerate the GTPase activity of Ras proteins $(19,20)$ and is in contrast to the results obtained with the second IRAI disruption, farther upstream at codon 1058. This disruption (TM101) produced membranes with strikingly low levels of adenylate cyclase activity compared with wild-type or TM105 membranes.

Comparison of membrane and cytosol fractions showed that the majority of adenylate cyclase activity in wild-type strain HR125 was associated with the membrane fraction $(80 \%)$ and only a small portion $(20 \%)$ was located in the soluble fraction (Fig. 1C). This distribution remained unchanged in TM105, carrying the IRAl disruption at codon 2520. However, this pattern changed dramatically in cells carrying the disruption at codon 1058 (TM10i). In these cells, ädenylate cyclase activity was found almost entirely in the soluble fraction $(90 \%)$; only a small portion $(10 \%)$ was associated with the membrane fraction. The total activity in the TM101 fractions was always lower than that found in wild-type fractions; membrane specific activity was typically reduced by approximately 200 -fold, to barely measurable levels. The specific activity of the cytosolic fraction was also reduced, but by only a factor of 2: from an average of $0.70 \mathrm{U}$ (picomoles of cAMP per minute) per $\mathrm{mg}$ to an average of 


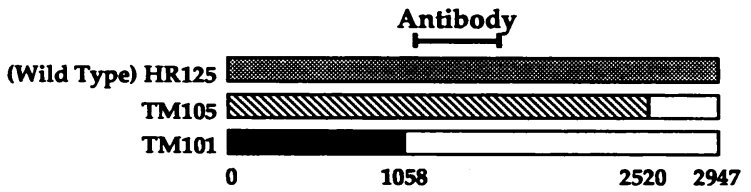

A.

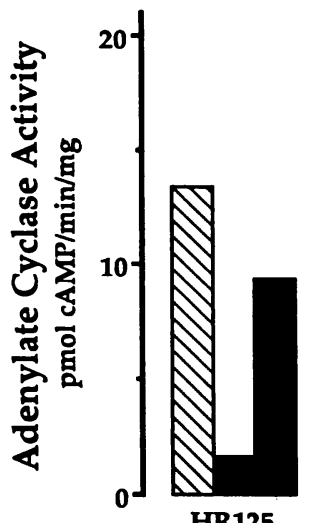

HR125
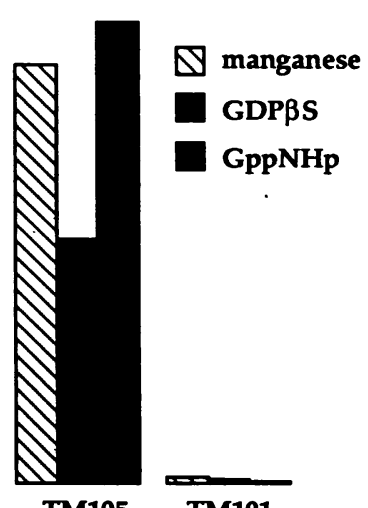

B.

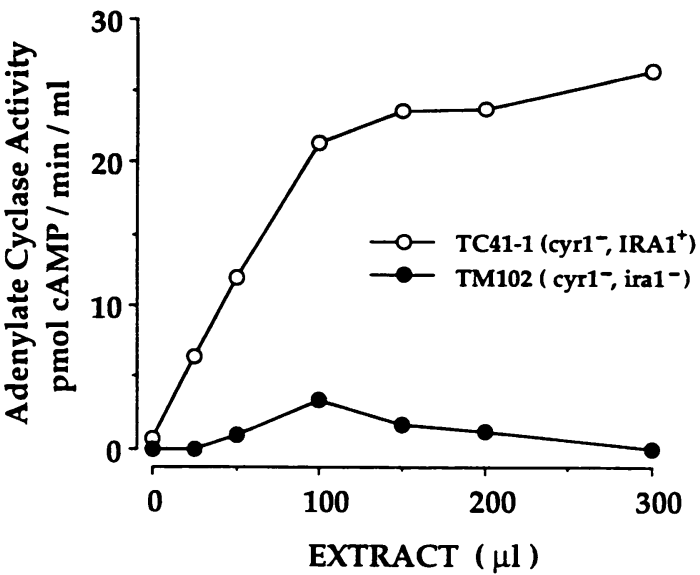

FIG. 2. Evidence that disruption of IRAI abolishes the capacity of membranes to bind adenylate cyclase. Membranes from wild-type strain $381 \mathrm{G}(17.6 \mathrm{mg})$ were extracted with $1.0 \mathrm{ml}$ of YMB containing $500 \mathrm{mM} \mathrm{NaCl}$ as described in the text, and the extract containing adenylate cyclase activity was collected. Samples containing the indicated amounts of extract were mixed with $5 \mathrm{mg}$ of acceptor membranes prepared from cyrl strains (with no adenylate cyclase activity). Reconstitution of adenylate cyclase to the acceptor membranes was initiated by diluting $\mathrm{NaCl} 10$-fold, and the mixture was incubated on ice for $30 \mathrm{~min}$. The membranes were collected by centrifugation and assayed for adenylate cyclase activity in the presence of $\mathrm{Mn}^{2+}$. Values plotted represent specific binding, obtained by subtracting the activity of control samples containing no acceptor membranes. Acceptor membranes used were from a cyrl strain with either a wild-type IRAI (TC41-1) or from TM102, a cyrl strain carrying the IRAl disruption at codon 1058 as shown in Fig. 1A for strain TM101.

have examined the shift from the membrane to the cytosol in more detail.

IRAI disruption prevents membrane binding of adenylate cyclase. A reconstitution assay revealed that the $I R A I$ disruption at codon 1058 produced membranes that had lost the ability to bind adenylate cyclase. Adenylate cyclase activity can be extracted from wild-type membranes with $0.5 \mathrm{M}$ $\mathrm{NaCl}$ and then reconstituted onto membranes prepared from a cyrl yeast strain (TC41-1) lacking adenylate cyclase activity. We have previously used this type of reconstitution assay to measure the capacity of membranes to bind adenylate cyclase (15). Such a reconstitution experiment, comparing membranes from IRAI-disrupted cells with membranes from cells carrying a normal IRAI gene, is shown in Fig. 2. In this experiment, acceptor membranes were incubated with various concentrations of salt-extracted adenylate cyclase from wild-type cells and the $\mathrm{NaCl}$ concentration was then lowered by dilution to allow binding of the enzyme to the membranes. Membranes were then collected by centrifugation and assayed for reconstituted adenylate cyclase activity. Values plotted represent specific binding, obtained by subtracting the activity of control samples containing no acceptor membranes. The activity in these control samples averaged approximately $20 \%$ of that obtained with the TC41-1 samples (15).

Membranes prepared from cyrl yeast cells with a wildtype IRAI gene (TC41-1) bound increasing amounts of adenylate cyclase activity as the concentration of extract increased. In contrast, membranes prepared from a cyrl strain carrying the IRAI disruption at codon 1058 (TM102) failed to bind adenylate cyclase activity. This experiment 

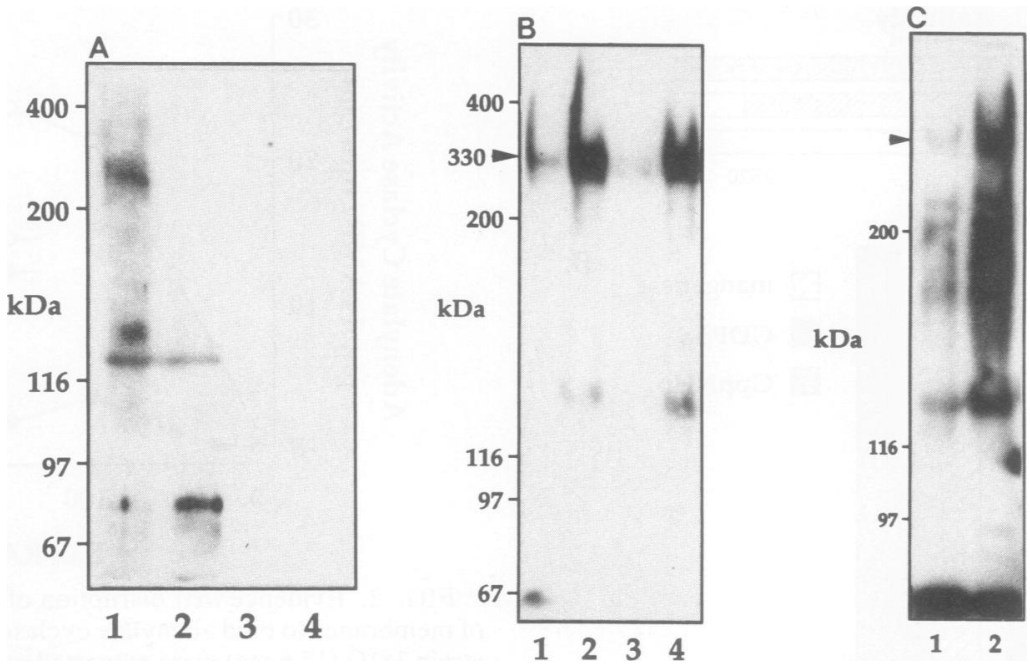

FIG. 3. Western blots with IRA1 antibodies. Antibodies were prepared against a $\beta$-galactosidase/IRA1 fusion protein, expressing amino acids 1164 to 1618 of IRA1 as indicated in Fig. 1A. (A) Yeast membranes (25 $\mu$ g per lane) from either wild-type cells (HR125; lanes 1 and 3) or cells carrying a disrupted IRAI (TM101; lanes 2 and 4) were run out a 4\% SDS-gel and transferred to nitrocellulose. Blots were developed with either preimmune (lanes 3 and 4) or immune (lanes 1 and 2) serum, diluted 1:600, and ${ }^{125}$ I-labeled second antibody. (B) Wild-type membranes were detergent extracted with YMB containing $2 \%$ taurocholate, $1 \mathrm{mM} \mathrm{Ca}^{2+}$, and $1 \mathrm{mM} \mathrm{Mn}^{2+}$, and samples of the extract were incubated with either Sepharose 4B (lanes 1 and 3) or ConA-Sepharose (lanes 2 and 4) for $4 \mathrm{~h}$ at $4^{\circ} \mathrm{C}$. The resin samples were then washed with either YMB (lanes 1 and 2) or YMB with $0.5 \mathrm{M} \mathrm{NaCl}$ added (lanes 3 and 4). Proteins were then eluted with $1 \%$ SDS-5\% $\beta$-mercaptoethanol-5 mM EDTA at $65^{\circ} \mathrm{C}$. Samples were run on a $4 \%$ SDS-polyacrylamide gel and for Western blotting with IRA1 antibodies as described for panel A. (C) Samples were incubated with lentil lectin-agarose and analyzed by Western blotting as for panel B. Lanes: 1 , methyl- $\alpha$-D-mannopyranoside and methyl- $\alpha$-D-glucopyranoside added to a concentration of $60 \mathrm{mM}$ each; 2 , no competing sugars added.

indicated that the disruption of IRAI produced membranes lacking binding sites for adenylate cyclase activity. An alternate possibility was that the mutant membranes bound adenylate cyclase but inhibited the activity of the enzyme. However, the total activity observed in these experiments was the same with IRAI-disrupted membranes and wild-type membranes, indicating that the TM102 membranes did not inhibit adenylate cyclase activity.

Antibodies against IRA1 recognize a 330-kDa membrane protein. We have developed polyclonal antibodies against an IRA1/ $\beta$-galactosidase fusion protein (described in Materials and Methods). Western blots show that these antibodies recognize a 320 - to $350-\mathrm{kDa}$ protein matching the size predicted by the IRA1 amino acid sequence. This band was observed in samples from wild-type yeast membranes (Fig. $3 \mathrm{~A}$, lane 1); however, no immunoreactive bands were observed with IRAI-disrupted membranes (TM101; lane 2). This result was expected, since the IRAI disruption in TM101 is upstream of the portion of the IRA1 protein that the antibodies were raised against. No immunoreactive bands were seen with preimmune serum (lanes 3 and 4). Immunoreactive proteins were found only associated with membrane fractions, not in cytosolic fractions. We were unable to extract the IRA1 protein from membranes with $\mathrm{NaCl}$; however, some of the $330-\mathrm{kDa}$ protein was solubilized with $5 \%$ taurocholate.

IRA1 in detergent extracts bound to ConA (Fig. 3B), indicating that IRA1 is glycosylated. Detergent extracts of wild-type membranes were incubated with either ConASepharose or underivatized Sepharose 4B as a control. The resin was washed with buffer, and bound proteins were removed by treatment with reducing SDS-gel sample buffer at $65^{\circ} \mathrm{C}$. The proteins were then separated with SDS-PAGE for Western blotting with IRA1 antibodies. A small amount of IRA1 was associated with the control Sepharose 4B (Fig.
$3 \mathrm{~B}$, lane 1), but this decreased when $0.5 \mathrm{M} \mathrm{NaCl}$ was added to the buffer (lane 3). In contrast, a much more intense IRA1 signal was associated with the ConA-Sepharose (lanes 2 and 4). IRA1 also bound to lentil lectin-agarose (Fig. 3C). Lentil lectin has sugar specificity similar to that of ConA, and binding to the lectin resin was inhibited by the addition of methyl- $\alpha$-D-mannopyranoside and methyl- $\alpha$-D-glucopyranoside as competitive ligands (Fig. 3C, lane 1). Thus, on the basis of its diffuse appearance on gels and its interactions with two different lectins, IRA1 appears to be glycosylated.

Glycosylation of IRA1 indicates at least one extracellular domain. The IRA1 protein sequence contains numerous sites for $\mathrm{N}$-linked glycosylation as well as potential transmembrane regions (Fig. 4). IRA1 contains 34 potential sites for $\mathrm{N}$-linked glycosylation, half of which are found in two clusters in the first 900 residues. A Kyte-Doolittle hydrophobicity plot using a window of 19 amino acids identified five potential transmembrane domains in which the hydrophobic index exceeded 1.6; the locations and sequences of these segments are indicated in Fig. 4. Several other stretches of hydrophobic sequence came close to this value and may also be considered as potential transmembrane sequences (Fig. 4).

IRA1 antibodies inhibit membrane binding of adenylate cyclase. In a reconstitution experiment similar to that described for Fig. 2, antibodies to IRA1 inhibited the binding of adenylate cyclase activity to acceptor membranes (Fig. 5). When acceptor membranes from a strain lacking adenylate cyclase activity were incubated with antibodies against IRA1 prior to use in a reconstitution assay, adenylate cyclase binding was inhibited by approximately $50 \%$. We have been unable to completely inhibit reconstitution with our antibodies. The fact that these IRA1 antibodies did not entirely inhibit reconstitution suggests the possibility of a second class of IRA1 binding sites, not recognized by the antibod- 


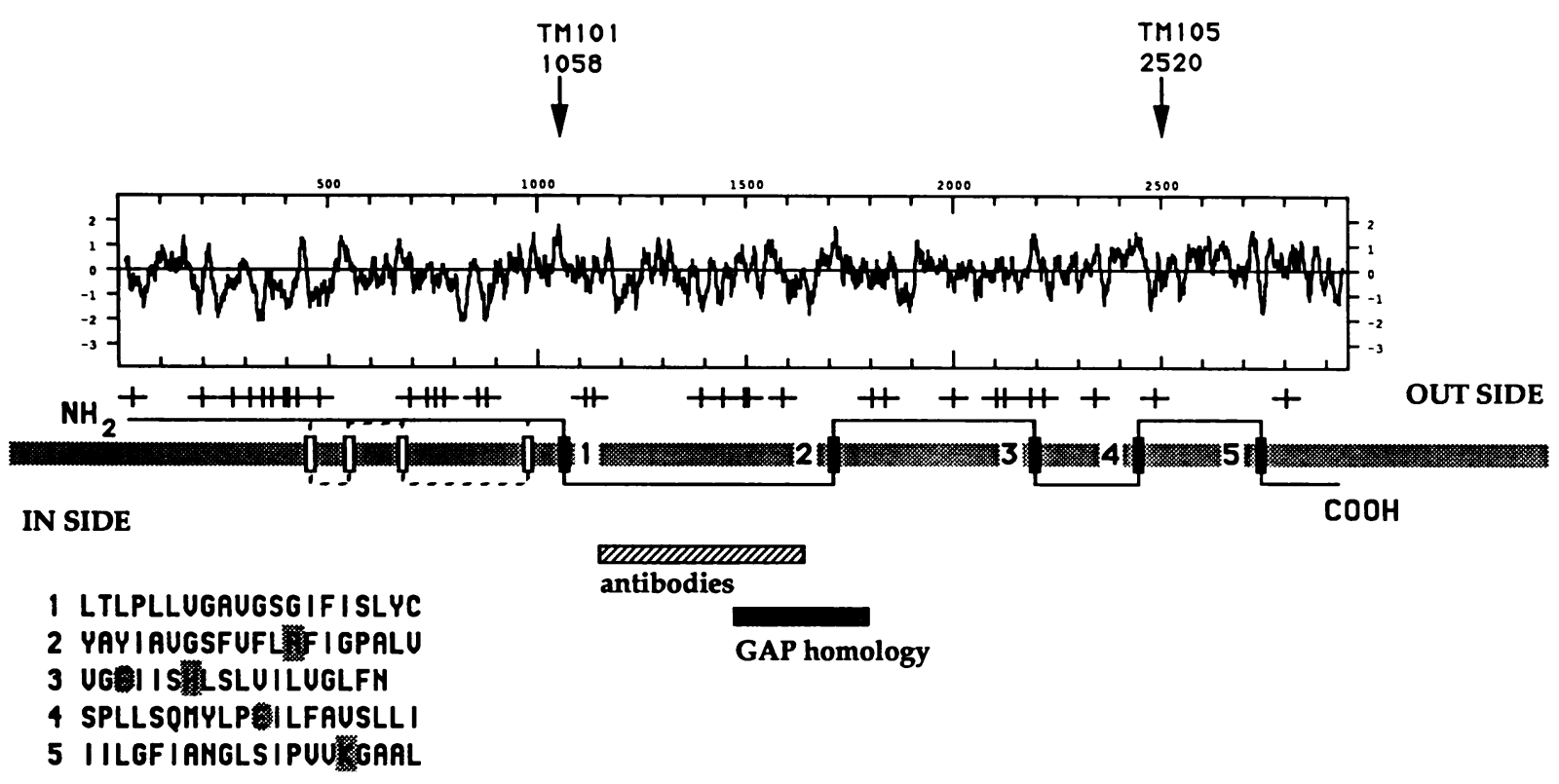

FIG. 4. Potential membrane-spanning domains in IRA1. A Kyte-Doolittle hydropathy plot for the predicted amino acid sequence is aligned with the positions of the disruptions in mutants TM101 and TM105, the region used for antibody production, and the region of GAP homology, as indicated. A schematic of the membrane (thick shaded line) shows the position of potential membrane-spanning regions attaining a score of 1.6 or greater. These are represented as numbered shaded bars across the membrane, and the sequences of these regions are presented next to the respective numbers. Additional potential transmembrane domains are indicated as unshaded bars, and two potential topologies are represented by the narrow lines that traverse the membrane. Potential $\mathrm{N}$-linked glycosylation sites are also indicated $(+)$.

ies. The antiserum had no direct effect on adenylate cyclase activity, and preimmune serum did not inhibit reconstitution of adenylate cyclase activity (not shown).

Comigration of IRA1 and adenylate cyclase. In detergent solution, adenylate cyclase activity and the IRA1 protein comigrated on a Sepharose 4B gel filtration column. Wildtype (381G) membranes were extracted with $5 \%$ taurocholate, and the extract was applied to a Sepharose 4B

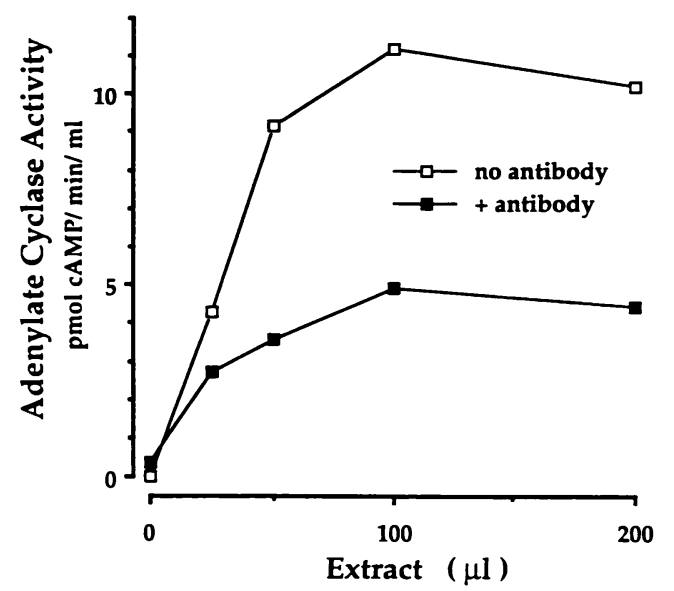

FIG. 5. Inhibition of reconstitution by IRA1 antibodies. Samples were prepared for reconstitution as described for Fig. 2 except that the acceptor membranes (TC41-1;5 mg) were pretreated either with antiserum against IRA1 $(10 \mu \mathrm{l})$ or with reconstitution buffer (no antibody) for $4 \mathrm{~h}$ on ice prior to use in the reconstitution. Values plotted represent specific binding, corresponding control samples containing no TC41-1 membranes; values obtained were subtracted from the experimental data as described for Fig. 2. column. Fractions were collected and assayed for adenylate cyclase activity. Samples from these fractions were also run on a 4\% SDS-gel for Western blotting with IRA1 antibodies. Figure 6 allows comparison of the elution positions of adenylate cyclase activity (Fig. 6B) and the 330-kDa IRA1 protein (Fig. 6A). At low ionic strength, both adenylate cyclase and IRA1 protein levels peaked in the fraction immediately following the void volume, indicating a large complex. When $0.5 \mathrm{M} \mathrm{NaCl}$ was added to the membrane extract and the column buffer, both adenylate cyclase and IRA1 migrated as smaller particles, and they no longer migrated with the same elution profile.

We interpret these results to indicate that at low ionic strength, IRA1 and adenylate cyclase are physically associated. However, an alternate interpretation cannot be discounted: the proteins may comigrate by chance. IRA1 and adenylate cyclase may be independently associated with separate large complexes that are dissociated by high ionic strength. As a more specific test of association between IRA1 and adenylate cyclase, we measured the size of the IRA1 complex in both the presence and absence of adenylate cyclase. If IRA1 is associated with adenylate cyclase, then the size of the complex containing IRA1 should be reduced in the absence of adenylate cyclase. As shown in Fig. 7, we have found this to be the case. Detergent extracts were prepared from three different strains, a wild-type strain (HR125), a strain carrying a deleted $C Y R I$ gene (TC41-1), and a strain carrying $C Y R 1$ on YEp24, a multicopy plasmid (HR125::pAC2). TC41-1 has no adenylate cyclase activity or protein, and HR125::pAC2 expresses levels of adenylate cyclase approximately fivefold higher than those of the isogenic wild-type strain. The extracts were fractionated on the Sepharose 4B column, and the elution position of IRA1 was measured by Western blotting samples from each fraction. Figure 7A shows the $330-\mathrm{kDa}$ regions of the Western 


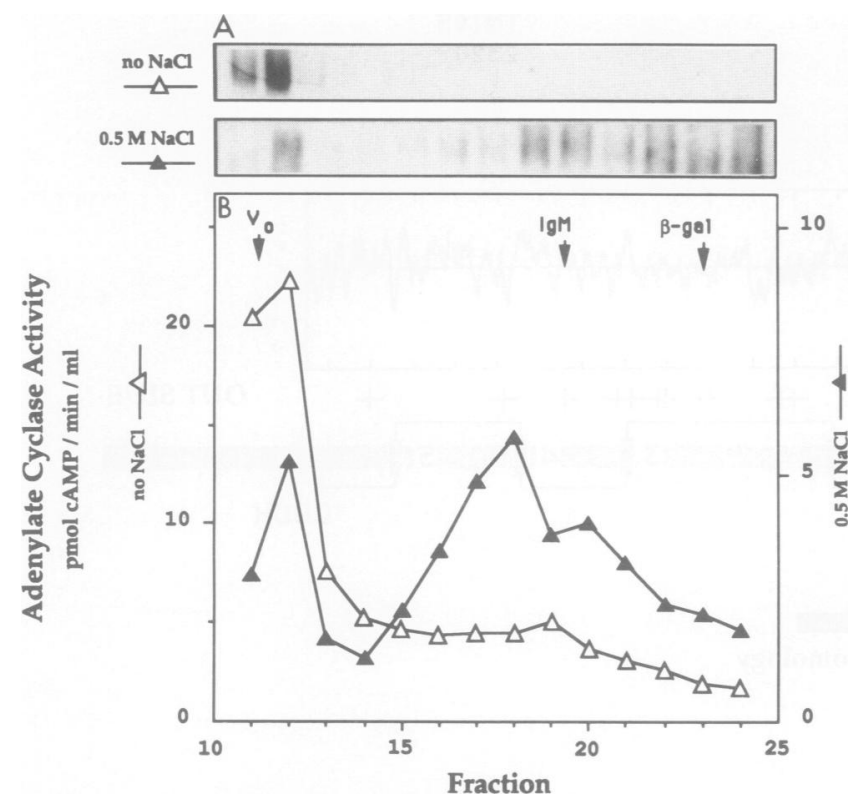

FIG. 6. Cochromatography of IRA1 with adenylate cyclase activity at low ionic strength. Detergent extracts containing adenylate cyclase activity were prepared by incubating wild-type (381G) membranes $(15 \mathrm{mg})$ in $1.4 \mathrm{ml}$ of YMB with $5 \%$ taurocholate on ice for $1 \mathrm{~h}$. The mixture was then centrifuged for $15 \mathrm{~min}$ at $14,000 \mathrm{rpm}$ $(16,000 \times g)$, and the supernatant $(1 \mathrm{ml})$ was loaded onto a Sepharose 4B column (1 by $46 \mathrm{~cm}$ ) equilibrated in $50 \mathrm{mM}$ MES-250 $\mathrm{mM}$ sucrose $-5 \mathrm{mM} \mathrm{MgCl}_{2}-1 \mathrm{mM}$ EDTA- $-0.02 \%$ sodium azide- $-0.5 \%$ sodium taurocholate. Fractions $(1.1 \mathrm{ml})$ were collected for adenylate cyclase assay with $\mathrm{Mn}^{2+}$ (open triangles). In a parallel experiment, $\mathrm{NaCl}(0.5 \mathrm{M})$ was added to both the extract and column equilibration buffer prior to chromatography (closed triangles). Samples $(100 \mu \mathrm{l})$ were also run on $4 \%$ SDS-gels for Western blotting with antibodies to IRA1 as described for Fig. 3. (A) The 330-kDa regions from the indicated blots; (B) relative density of each band.

blots, and Fig. 7B shows scanning densitometer measurements of each band. In the absence of adenylate cyclase, (strain TC41-1), practically all of the IRA1 protein migrated well within the included volume of the column, while in the wild-type extracts, a significant fraction of the IRA1 protein eluted near the void volume, indicating the formation of a larger complex. The proportion of IRA1 travelling as the large complex was increased even further in the extract from HR125::pAC2 cells, which produce elevated levels of adenylate cyclase. The elution position of IRA1 in the absence of adenylate cyclase matched that observed in wild-type cell extracts run at high ionic strength.

\section{DISCUSSION}

Our results support a model in which adenylate cyclase and the IRA1 polypeptide are associated with each other. This conclusion is based on multiple lines of biochemical and genetic evidence. We propose that IRAl plays a membraneanchoring role for adenylate cyclase in addition to its previously known regulatory role (18-20).

Effect of IRAI disruptions on adenylate cyclase activity. Previous disruptions of the IRAl gene, including the disruption at codon 2520 in TM105, have produced a phenotype characterized by heat shock sensitivity and elevated cAMP levels (18). This phenotype is characteristic of mutations that activate the Ras/adenylate cyclase pathway. In our hands,

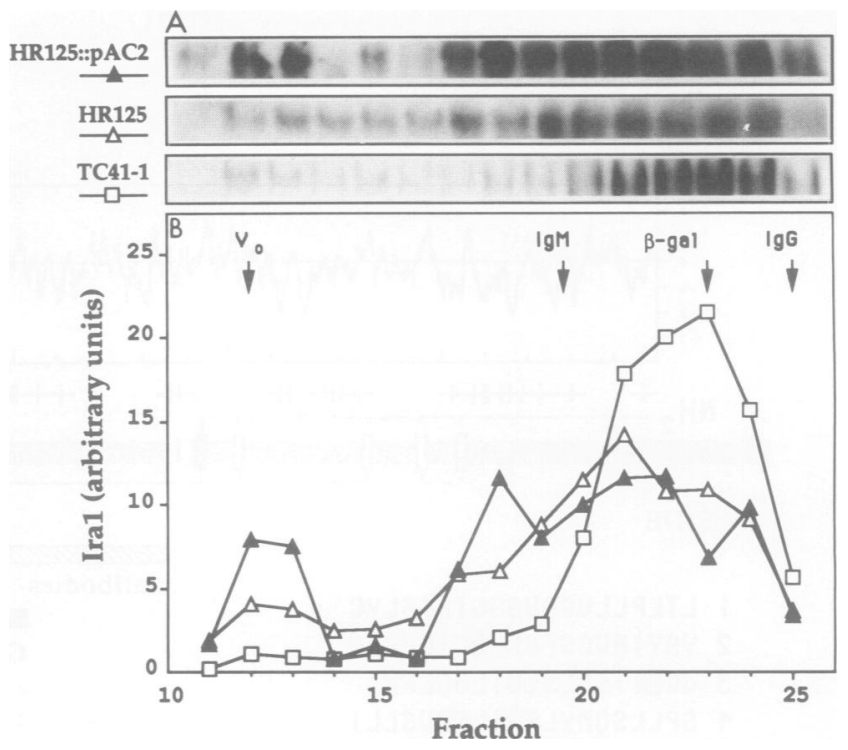

FIG. 7. Sepharose 4B chromatography of IRAl in the absence of adenylate cyclase. Detergent extracts from the indicated strains were prepared for chromatography at low ionic strength as described for Fig. 5. HR125::pAC2 overexpresses adenylate cyclase, HR125 is a wild-type strain, and TC41-1 has had the structural gene for adenylate cyclase $(C Y R I)$ deleted. Fractions were collected, and samples $(100 \mu \mathrm{l})$ were run on $4 \%$ SDS-gels for Western blotting with antibodies to IRA1 as described for Fig. 3. (A) The 330-kDa regions from the blots; (B) relative density of each band, quantitated by scanning with a Molecular Dynamics computing densitometer. Density values are given as percentage of the total for each blot.

the codon 2520 mutation produced substantial elevation of basal adenylate cyclase activity. These observations are consistent with the reports that this disruption blocks the ability of IRA1 to accelerate the GTPase activity of Ras proteins (18). The disruption at codon 1058 (strains TM101 and TM102) produced an almost opposite phenotype. Rather than resembling cells with an activated Ras/adenylate cyclase system, these mutants appear deficient in cAMP production. These contrasting phenotypes can be reconciled by taking into account the change in subcellular location of adenylate cyclase in these mutants. Presumably, all of the IRAI disruptions produce activated Ras proteins, but the disruption at codon 1058 also releases adenylate cyclase from the membranes into the cytosol, where it can no longer be efficiently coupled to Ras. Thus, in our model, two functions can be assigned to IRA1: regulation of Ras GTPase activity and complex formation with adenylate cyclase. This interpretation is supported by the finding that membranes from mutants TM101 and TM102 have a greatly reduced capacity to bind adenylate cyclase in vitro. Some low level of binding must remain in the TM101 disruptants, since the cells grow, albeit slowly. They must therefore retain some limited ability to couple Ras to adenylate cyclase, since cells lacking Ras function will not grow at all.

Characterization of the IRA1 protein. Previous experiments using gel filtration to measure the size of taurocholatesolubilized adenylate cyclase have shown that the enzyme can exist as two different-size complexes. At low ionic strength, adenylate cyclase behaves as a very large particle of several million daltons. However, at higher ionic strength $(0.5 \mathrm{M} \mathrm{NaCl})$, this complex dissociates, and the enzyme 
migrates as a smaller particle of approximately $600 \mathrm{kDa}$. Adenylate cyclase can also be dissociated from this complex, without prior detergent solubilization, by extracting membranes with $\mathrm{NaCl}$. The salt-extracted enzyme is indistinguishable from the $600-\mathrm{kDa}$ complex seen in salt-treated detergent extracts. Thus, $\mathrm{NaCl}$ appears to dissociate adenylate cyclase from a complex that anchors the enzyme to the membrane.

If IRA1 is the membrane anchor for adenylate cyclase, then we should observe the two proteins comigrating at low ionic strength, where adenylate cyclase is associated with the complex, but not at high ionic strength. This is what we have observed; at low ionic strength, the peaks of both IRA1 and adenylate cyclase activity eluted in the fraction immediately following the void volume. Although these proteins eluted in the included volume, the resolution of the column in this region is very limited. The proteins could be fortuitously travelling together. Therefore, although this result is consistent with our model, it falls short of demonstrating association between adenylate cyclase and IRA1.

The experiments shown in Fig. 7, demonstrating that adenylate cyclase contributes to the size of the IRA1 complex, provide more compelling evidence indicating association between the two proteins. In wild-type extracts, or in extracts prepared from cells overexpressing adenylate cyclase, the elution position of some of the IRA1 is shifted to that of the larger particle. In extracts prepared from cells lacking adenylate cyclase, IRA1 elutes well in the included volume as a particle similar in size to $\beta$-galactosidase (520 $\mathrm{kDa})$. The elution pattern of IRA1 in the absence of adenylate cyclase is similar to the elution position of IRA1 in wild-type extracts at high ionic strength. Though dissociated from the larger complex, even the smaller form of IRA1 migrates as a particle larger than expected for monomeric IRA1. This could represent a very elongated IRA1 monomer or IRA1 involved in some sort of oligomeric structure.

The proportion of IRA1 eluting as the large complex in the wild-type (381G) IRA1 elution profiles shown in Fig. 6 (low ionic strength) is greater than that shown in Fig. 7 (HR125). The profile from Fig. 6 shows almost all of the IRA1 protein migrating as the large form, indicating that in this extract all of the IRA1 is involved in some large oligomeric complex; in Fig. 7, it appears that a smaller proportion of the IRA1 protein migrates as the larger complex. Some of this difference may be due to strain-to-strain variation in adenylate cyclase activity. We used wild-type strain $381 G$ in the experiment shown in Fig. 6 because it has high levels of adenylate cyclase activity and because this activity is easily extracted from the membrane. Thus, $381 \mathrm{G}$ is more suitable for experiments following adenylate cyclase activity through column chromatography. We have used strain HR125 (Fig. 7) for genetic work. HR125 is lower in adenylate cyclase activity than $381 \mathrm{G}$, even in the isogenic strain overexpressing CYRI (HR125::pAC2). Beyond this difference between strains, we have observed some variation in IRA1 levels between membrane preparations. The variations in the levels of these two proteins may account for some of the changes in the amount of IRA1 that migrates as the larger complex.

IRA1 structure. The lectin-binding experiments indicate that IRA1 is glycosylated and is therefore likely to traverse the plasma membrane at least once. As indicated by the Kyte-Doolittle hydropathy plot in Fig. 4, there are several candidates for transmembrane sequences in the IRA1 predicted amino acid sequence $(9,11)$. Five regions meet the criterion of reaching a peak value of at least 1.6 when averaged over a window of 19 amino acids. Additional transmembrane regions that do not quite reach an index of 1.6 can be postulated; four of these are indicated in Fig. 4 . IRA2 also has peaks of hydrophobicity in these regions. Clearly the actual number of transmembrane segments will be determined only by much more thorough experimentation; however, with the evidence at hand, we can begin to make a preliminary model of IRA1 membrane topology. Such a model will be of value in developing tests to further refine our picture of IRA1 structure. $\mathrm{N}$-linked glycosylation sites are clustered in several regions spread across the sequence (Fig. 4); however, there is an unusually high concentration of potential glycosylation sites at the amino end of the protein, with 11 sites located within the first 500 amino acids. We therefore propose that the amino terminus is probably on the outside of the membrane.

Tanaka et al. (18) have reported a disruption of IRAI at codon 1430 that produces the activated Ras phenotype. It is therefore likely that adenylate cyclase in these mutants remains coupled to Ras and is associated with the membrane. This indicates that some structure between position 1430 and our disruption at codon 1058 is important for proper localization of adenylate cyclase. It is interesting that our antibodies that block adenylate cyclase binding also recognize this region. These antibodies were raised against a fusion protein expressing amino acids 1127 to 1618 of IRA1. Since the interaction between IRA1 and adenylate cyclase must take place within the cell, this region of IRA1 likely occupies the cytoplasmic face of the membrane. Additionally, the nearby region of GAP homology, occupying positions 1451 to 1780 , must interact with the cytoplasmic Ras proteins and is also likely to be located in the interior of the cell. A model of IRA1 membrane topology based on these constraints is shown in Fig. 4. It should be emphasized that there are alternative interpretations of the data that lead to different models of IRA1 structure. In particular, the actual number of membrane-spanning domains will have a large impact on the model.

IRA1/adenylate cyclase complex. Our results indicate that IRA1 and adenylate cyclase are associated in a large oligomeric complex. This view is based on the results obtained with mutations, antibodies, and gel filtration chromatography. If such a complex exists, what proteins are included? Presumably the complex includes at least the CYR1 catalytic subunit, IRA1, and cyclase-associated protein (also identified as the product of the $S R V 2$ gene) $(6,7)$. Ras proteins must also be associated with the complex, at least on a transient basis. Are there other proteins involved? By virtue of physical and functional similarities to IRA1, the IRA2 gene product is a good candidate to serve a function similar to that of IRA1. Inclusion of IRA2 in the complex might explain several observations. Antibodies against IRA1 were effective in blocking only half of the membrane capacity to bind adenylate cyclase, while disruption of IRAI almost entirely abolished binding of adenylate cyclase to the membrane. This finding might be explained by a second class of binding sites (such as IRA2) that is not blocked by the antibodies but is dependent on a functional IRA1 protein for stability. The disruption of $I R A 1$ at codon 1058 may have decreased the stability of all of the members of the complex. In the absence of adenylate cyclase, IRA1 migrates as a particle of approximately twice the size expected for monomer IRA1. This is approximately the size that would be expected of an IRA1/IRA2 dimer.

Role of IRA1 in regulating CAMP production. Association between IRA1 and adenylate cyclase implies that Ras pro- 
teins interact with both adenylate cyclase and IRA1 either simultaneously or in quick succession. The fact that IRA1 turns off Ras activity indicates that termination of Ras activation does not begin until the Ras protein encounters the enzyme complex (unless there is a substantial excess of IRA1). This would prevent a futile cycle of Ras proteins being activated and inactivated without finding their target.

To date, IRA1 is the only protein identified in the Ras/ adenylate cyclase pathway that appears to span the membrane. Although a transmembrane protein is not absolutely necessary, such a protein would be well suited for receiving signals originating outside of the cell. Perhaps related to this model is the finding that the smaller GAP, which is cytoplasmic, interacts with a transmembrane mitogen receptor, the platelet-derived growth factor receptor (10). The model that we have described allows more regulatory input into the Ras system. Multiple inputs are appropriate to a system that must regulate growth in response to many different environmental conditions, a need that is held in common between yeast and animal cells.

\section{ACKNOWLEDGMENTS}

We thank Henry R. Bourne for reading the manuscript, Kazuma Tanaka for providing plasmid pNR1, and G. Garcia for help in preparing cell extracts.

This work was supported by the Public Health Service grant GM42406-01 from the National Institutes of Health.

\section{REFERENCES}

1. Barbacid, M. 1987. ras genes. Annu. Rev. Biochem. 56:779827.

2. Botstein, D., S. C. Falco, S. C. Stewart, M. Brennan, S. Scherer, D. T. Stinchcomb, K. Struhl, and R. W. Davis. 1979. Sterile host yeasts (SHY): a eukaryotic system of biological containment for recombinant DNA experiments. Gene 8:17-24.

3. Buchberg, A. M., L. S. Cleveland, N. A. Jenkins, and N. G. Copeland. 1990. Sequence homology shared by neurofibromatosis type-1 gene and $I R A-I$ and $I R A-2$ negative regulators of the $R A S$ cyclic AMP pathway. Nature (London) 347:291-294.

4. Casperson, J. F., N. Walker, and H. R. Bourne. 1985. Isolation of the gene encoding adenylate cyclase in the yeast Saccharomyces cerevisiae. Proc. Natl. Acad. Sci. USA 82:5060-5063.

5. Casperson, J. F., N. Walker, A. R. Brasier, and H. R. Bourne. 1983. A guanine nucleotide sensitive adenylate cyclase in the yeast Saccharomyces cerevisiae. J. Biol. Chem. 258:7911-7914.

6. Fedor-Chaiken, M., R. J. Deschenes, and J. R. Broach. 1990 SRV2, a gene required for RAS activation of adenylate cyclase in yeast. Cell 61:329-340.

7. Field, J., A. Vojtek, R. Ballester, G. Bolger, J. Colicelli, K. Ferguson, J. Gerst, T. Kataoka, T. Michaeli, S. Powers, M. Riggs, L. Rodgers, I. Wieland, B. Wheland, and M. Wigler.
1990. Cloning and characterization of CAP, the S. cerevisiae gene encoding the $70 \mathrm{kd}$ adenylyl cyclase-associated protein. Cell 61:319-327.

8. Heideman, W., G. F. Casperson, and H. R. Bourne. 1987. Adenylyl cyclase in yeast: hydrodynamic properties and activation by trypsin. J. Biol. Chem. 262:7087-7091.

9. Jähnig, F. 1990. Structure predictions of membrane proteins are not that bad. Trends Biochem. Sci. 15:93-95.

10. Kazlauskas, A., C. Ellis, T. Pawson, and J. A. Cooper. 1990. Binding of GAP to activated PDGF receptors. Science 247: 1578-1581.

11. Kyte, J., and R. F. Doolittle. 1982. A simple method for displaying the hydrophobic character of a protein. J. Mol. Biol. 157:105-132.

12. Lowry, O. H., N. S. Rosebrough, A. L. Farr, and R. J. Randall. 1951. Protein measurement with the Folin phenol reagent. J. Biol. Chem. 193:265-275.

13. Marsh, L. J., M. Erfle, and E. J. Wykes. 1984. The pIC plasmid and phage vectors with versatile cloning sites for recombinant selection by insertional inactivation. Gene 32:481-485.

14. McCormick, F. 1989. ras GTPase activating protein: signal transmitter and signal terminator. Cell 56:5-8.

15. Mitts, M. R., D. B. Grant, and W. Heideman. 1990. Adenylate cyclase in Saccharomyces cerevisiae is a peripheral membrane protein. Mol. Cell. Biol. 10:3873-3883.

16. Rothstein, R. J. 1983. One-step gene disruption in yeast. Methods Enzymol. 101:202-211.

17. Salomon, Y., C. Londos, and M. Rodbell. 1974. A highly sensitive adenylate cyclase assay. Anal. Biochem. 58:541-548.

18. Tanaka, K., K. Matsumoto, and A. Toh-e. 1989. IRAI, an inhibitory regulator of the $R A S$-cyclic AMP pathway in Saccharomyces cerevisiae. Mol. Cell. Biol. 9:757-768.

19. Tanaka, K., M. Nakafuku, T. Satoh, M. S. Marshall, J. Gibbs, K. Matsumoto, Y. Kaziro, and A. Toh-e. 1990. S. cerevisiae genes IRAI and Ira2 encode proteins that may be functionally equivalent to mammalian ras GTPase activating protein. Cell 60:803-807.

20. Tanaka, K., M. Nakafuku, F. Tamanoi, Y. Kaziro, K. Matsumoto, and A. Toh-e. 1990. IRA2, a second gene of Saccharomyces cerevisiae that encodes a protein with a domain homologous to mammalian GTPase-activating protein. Mol. Cell. Biol. 10: 4303-4313.

21. Trahey, M., and F. McCormick. 1987. A cytoplasmic protein stimulates normal N-ras p21 GTPase, but does not affect oncogenic mutants. Science 238:542-545.

22. Wallace, M., D. A. Marchuk, L. B. Andersen, R. Letcher, H. M. Odeh, A. M. Saulino, J. W. Fountain, A. Brereton, J. Nicholson, A. L. Mitchell, B. H. Brownstein, and F. S. Collins. 1990. Type 1 neurofibromatosis gene: identification of a large transcript disrupted in three NF1 patients. Science 249:181-186.

23. Xu, G., P. O'Connell, D. Viskochil, R. Cawthon, M. Robertson, M. Culver, D. Dunn, J. Stevens, R. Gesteland, R. White, and R. Weiss. 1990. The neurofibromatosis Type 1 gene encodes a protein related to GAP. Cell 62:599-608. 